\title{
An Efficient Analysis Method for Cylindrical Conformal Microstrip Antenna Fed by Microstripline
}

\author{
Chengyou Yin ${ }^{1}$ and Mengzhong $\mathrm{Hu}^{2}$ \\ ${ }^{1}$ Department of Information Engineering, Hefei Electronic Engineering Institute, 460 Huangshan Road, Anhui, Hefei 230037, China \\ ${ }^{2}$ Branch Team 91, Troops 92941, PLA, Liaoning, Huludao 125000, China
}

Correspondence should be addressed to Chengyou Yin, cyouyin@sina.com

Received 25 December 2011; Accepted 27 October 2012

Academic Editor: Hala A. Elsadek

Copyright ( $) 2012$ C. Yin and M. Hu. This is an open access article distributed under the Creative Commons Attribution License, which permits unrestricted use, distribution, and reproduction in any medium, provided the original work is properly cited.

\begin{abstract}
Firstly, a domain-division solution is provided in this paper, which can solve Hankel function's singular problem of Sommerfeld integral in calculating the spatial domain Green function for cylindrically stratified media and realize the computation for all elements of $Z$-matrix in the method of moment. Then, the arbitrary shape cylindrical conformal microstrip antenna (CCMA) fed by microstripline is theoretically analysed using the RWG basis function. Numerical results are presented in the form of mutual coupling between two current modes and electromagnetic characteristics of a CCMA fed by microstripline. The results show that, comparing with the planar counterpart microstrip antenna, the reflection coefficient of the CCMA increases, the current on the microstripline varies sharply, and the backward radiation field appears. However, the frontward radiation field of the CCMA is similar to the planar case.
\end{abstract}

\section{Introduction}

Microstrip antennas find many applications in satellite and mobile communication because of their many advantages, such as low profile, light weight, easy fabrication, and conformability to mounting hosts. With the development of technology, people find it is not enough to do researches only on planar microstrip antennas, so they begin to study and analyse conformal microstrip antennas, such as cylindrical, spherical, conical, or paraboloidal conformal microstrip antennas. This paper just focuses on cylindrical conformal microstrip antenna (CCMA). It is found that the development of analysis method for CCMA is not as mature as the planar case. At present, researchers usually use the transmission line model, cavity model [1-3], or their improved models to analyse CCMA in engineering projects. However, these models are only accurate for regular shape (such as rectangle, triangle, or circle) CCMA with thin substrate and cannot be applied to analyse arbitrary shape CCMA. As full-wave solutions are more accurate and applicable to many structures, some researchers begin to study the MoM-based solution for CCMA, such as [413 in recent years. Reading these references, one can find that the spatial domain Green function of cylindrically stratified media is the focused problem. A quasistatic images extraction technique has been proposed to solve the spatial domain Green function of electric field and mixed potential, respectively, in $[4,5]$, but when the source and observation points are located at the same radial distance from the axis of conductive cylinder, it is not valid along axial line (defined as $\rho=\rho^{\prime}$ and $\varphi=\varphi^{\prime}$ ). An eigenfunction solution of the Green function for arbitrary source and observation locations has been provided in [6-8]; however it needs a lot of computer resources, time consuming, and does not adapt to analyse big size or complex shape CCMA. The spatial and spectral MoM for CCMA is studied in [8-11], but all of them used the rooftop basis function which is not the general basis function compared with RWG for complex shape CCMA. In addition, recent relative MoMbased electromagnetic simulation software (such as IE3D, FEKO, or ADS) does not realize the calculation of Green's function of cylindrically stratified media too. Therefore, based on the former research works, this paper will provide a method which can analyse arbitrary shape CCMA because of the RWG basis function used in MoM. The paper is documented in the following structure. In Section 2, theory 
TABLE 1: The values of $e_{1}, e_{2}$, and $e_{3}$ for different $u v$.

\begin{tabular}{lccc}
\hline$u v$ & $e_{1}$ & $e_{2}$ & $e_{3}$ \\
\hline$z z$ & 1 & 0 & 0 \\
$\varphi z$ & 0 & 1 & 1 \\
$z \varphi$ & 0 & 1 & 1 \\
$\varphi \varphi$ & 0 & 2 & 0 \\
\hline
\end{tabular}

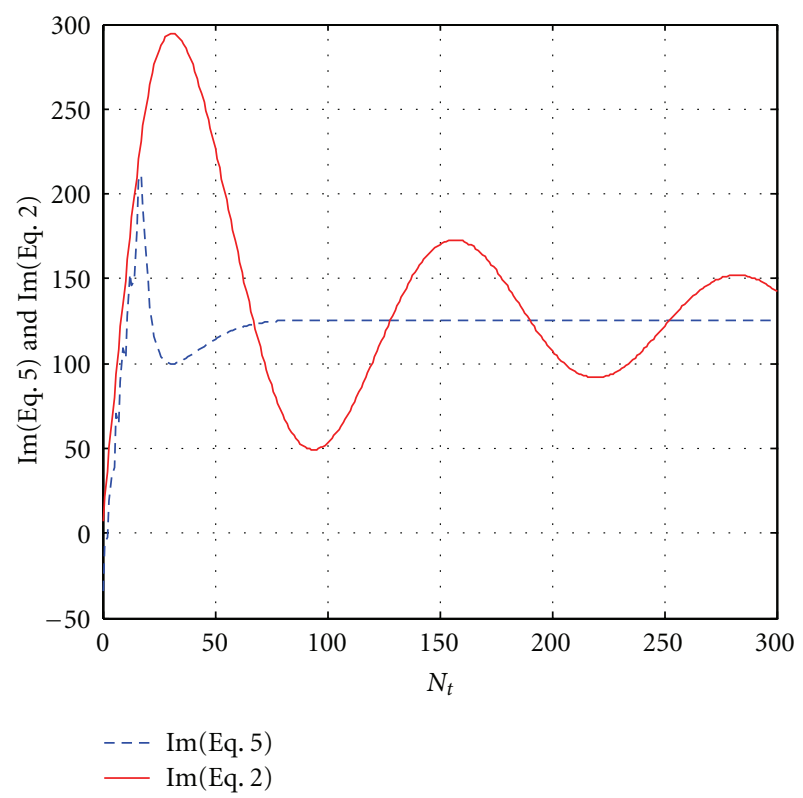

Figure 1: Comparison of $\widetilde{G}_{z z}^{E}$ imaginary part versus $N_{t}$ using (2) and (5).

and formulation of the domain-division solution for the Green's function for CCMA will be introduced in detail firstly. Then, based on RWG-MoM, theoretical analysis of arbitrary shape CCMA fed by microstripline will be done. In Section 3, a simulation example for investigating the effectiveness of the domain-division solution is presented and the electromagnetic characteristics of a CCMA fed by microstripline are gotten in another simulation example, in which results are compared with those of the planar case. A time convention of $e^{j \omega t}$ has been adopted in this paper.

\section{Theory and Formulation}

As we know, analysis of CCMA using MoM needs calculating the spatial Green function when source and observation points are located at the same radial distance from the axis of cylinder $\left(\rho=\rho^{\prime}\right)$, but, in this case, integral kernel function converges very slowly for electrically large conductive cylinders, because the Green's function involves Bessel and Hankel functions along with their derivatives. So the expressions of Green function gotten from $[12,13]$ cannot be directly used in the analysis of CCMA and some special work should be done. Combining the methods of [411], a domain-division solution can be provided to solve this problem which divides the computation of Green's function into two domain's problems. The first one is the unaxial line

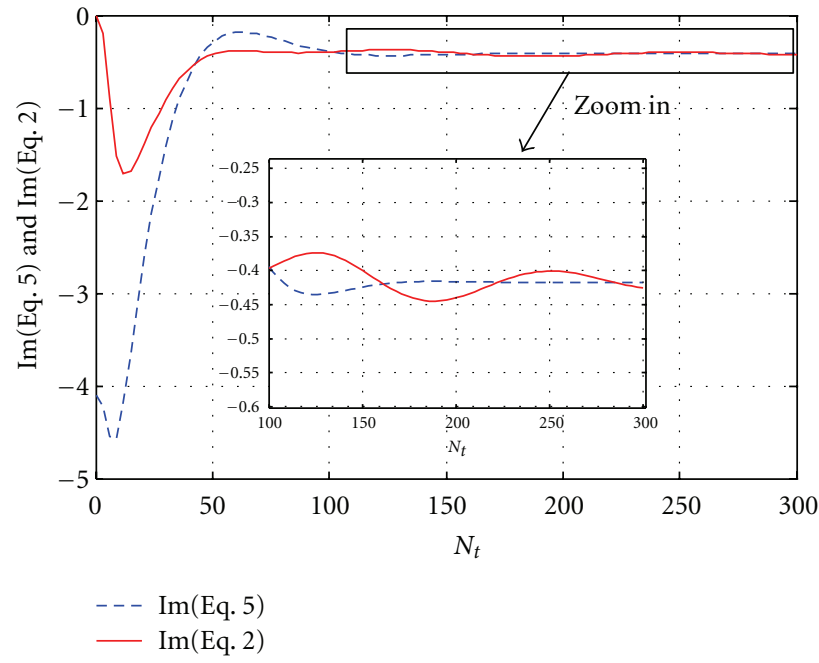

Figure 2: Comparison of $\widetilde{G}_{\varphi z}^{E}$ imaginary part versus $N_{t}$ using (2) and (5).

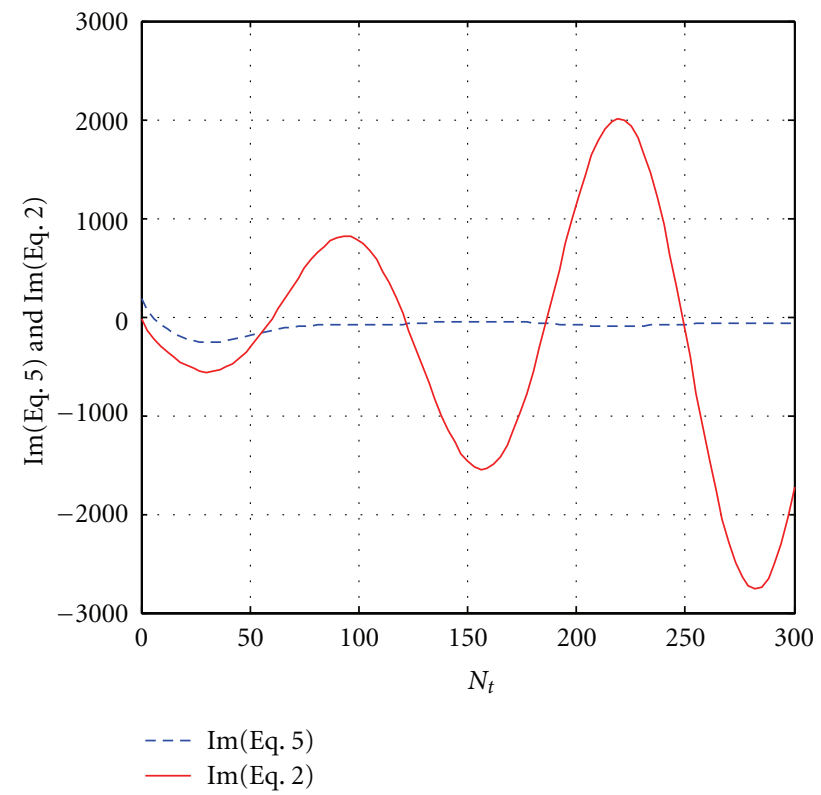

FIgURE 3: Comparison of $\widetilde{G}_{\varphi \varphi}^{E}$ imaginary part versus $N_{t}$ using (2) and (5).

problem (defined as $\left|\rho-\rho^{\prime}\right|=0$ and $\left|\varphi-\varphi^{\prime}\right|>0.05$ ), and the other one is the axial line problem (defined as $\left|\rho-\rho^{\prime}\right|=0$ and $\left.\left|\varphi-\varphi^{\prime}\right|<0.05\right)$.

2.1. Solution of the Unaxial Line Problem. Based on the formulation of spectral domain Green's function in [12, 13], the expressions of the spectral domain Green function components for the $n$th Harmonic wave can be rewritten as

$$
\frac{\tilde{G}_{u v}^{E_{n}}}{k_{z}^{e_{3}}}=\left(k_{\rho i}^{2}\right)^{e_{1}} n^{e_{2}} H_{n}^{(2)}\left(k_{\rho i} \rho\right) J_{n}\left(k_{\rho i} \rho^{\prime}\right) f^{u v}\left(n, k_{z}\right) .
$$

For different $u v$, the values of $e_{1}, e_{2}$, and $e_{3}$ in the above formula are show in Table 1. 


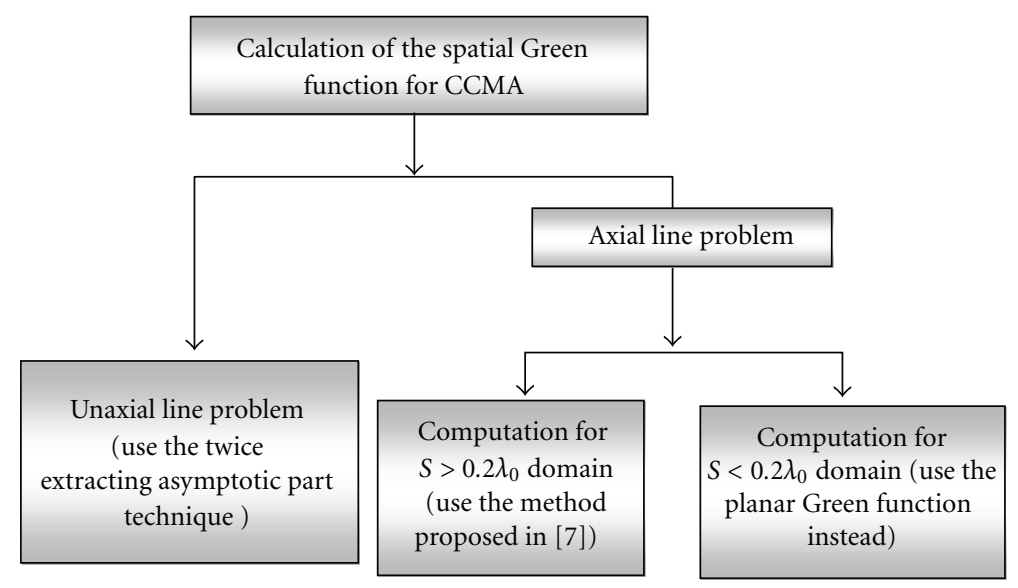

FIgURE 4: Calculation of the spatial Green function for MMCA.

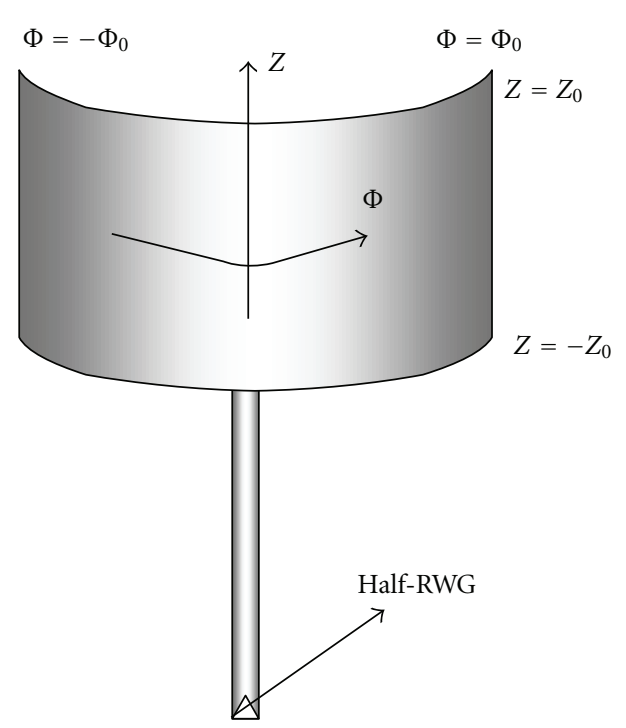

Figure 5: CCMA fed by microstripline.

Hence, the expressions of the spectral domain Green function for all tangential electric field components in cylindrical coordinates can be written as

$$
\begin{aligned}
\frac{\widetilde{G}_{u v}^{E}}{k_{z}^{e_{3}}}= & \frac{-1}{4 \omega} \sum_{n=-\infty}^{\infty}\left(k_{\rho i}^{2}\right)^{e_{1}} n^{e_{2}} H_{n}^{(2)}\left(k_{\rho i} \rho\right) J_{n}\left(k_{\rho i} \rho^{\prime}\right) \\
& \times f^{u v}\left(n, k_{z}\right) e^{j n\left(\varphi-\varphi^{\prime}\right)} .
\end{aligned}
$$

Using the expressions of the spectral domain Green function in $[12,13], f^{u v}$ ( $u$ and $v$ can be $z$ or $\varphi$ ) in the above formula is easy to get.

In (2), when $n \rightarrow \infty$, it is found that $f^{u v}\left(n, k_{z}\right)$ will converge to a function which is constant with respect to $n$,

$$
\lim _{n \rightarrow \infty} f^{u v}\left(n, k_{z}\right) \approx C_{u v}\left(k_{z}\right)
$$

With the aid of the series expression of $H_{0}^{(2)}\left(k_{\rho i}\left|\boldsymbol{\rho}-\boldsymbol{\rho}^{\prime}\right|\right)$, given by

$$
\sum_{n=-\infty}^{\infty} H_{n}^{(2)}\left(k_{\rho i} \rho\right) J_{n}\left(k_{\rho i} \rho^{\prime}\right) e^{j n\left(\varphi-\varphi^{\prime}\right)}=H_{0}^{(2)}\left(k_{\rho i}\left|\boldsymbol{\rho}-\boldsymbol{\rho}^{\prime}\right|\right),
$$

then (2) can be expressed as

$$
\begin{aligned}
\frac{\tilde{G}_{u v}^{E}}{k_{z}^{e_{3}}}= & \frac{-1}{4 \omega} \sum_{n=-\infty}^{\infty}\left(k_{\rho i}^{2}\right)^{e_{1}} n^{e_{2}} H_{n}^{(2)}\left(k_{\rho i} \rho\right) J_{n}\left(k_{\rho i} \rho^{\prime}\right) \\
& \times\left(f^{u v}\left(n, k_{z}\right)-C_{u v}\left(k_{z}\right)\right) e^{j n\left(\varphi-\varphi^{\prime}\right)} \\
& +\frac{-1}{4 \omega} C_{u v}\left(k_{z}\right)\left(k_{\rho i}^{2}\right)^{e_{1}} F_{1}^{u v}\left[H_{0}^{(2)}\left(k_{\rho i}\left|\rho-\boldsymbol{\rho}^{\prime}\right|\right)\right] .
\end{aligned}
$$

Expressions of $F_{1}^{u v}\left[H_{0}^{(2)}\left(k_{\rho i}\left|\rho-\rho^{\prime}\right|\right)\right]$ in (5) can be gotten using (4) and its derivatives, which can be found in [5]. Computation of $F_{1}^{u v}\left[H_{0}^{(2)}\left(k_{\rho i}\left|\boldsymbol{\rho}-\boldsymbol{\rho}^{\prime}\right|\right)\right]$ involves Bessel and Hankel functions' derivatives. With the aid of formula (1.2.34) in [14], their derivatives can be calculated easily.

From (5), one can find that the spectral domain Green function now includes two parts after extracting the asymptotic part with respect to $n$. The first part is still a summation of infinite series with respect to $n$, but it is now more rapidly convergent and the limits of the infinite summation can be truncated at relatively small values $N_{t}$. The second part is the asymptotic part of the spectral domain Green function which now is not an infinite summation but a function with respect to $H_{0}^{(2)}\left(k_{\rho i}\left|\boldsymbol{\rho}-\boldsymbol{\rho}^{\prime}\right|\right)$.

To study the convergence of formulae (2) and (5), the spectral domain Green function is computed by (2) and (5), respectively. The results are shown in Figures 1, 2, and 3.

Parameters used in the above computation are set as follow: $\Delta \varphi=0.05, a_{0}=3 \lambda_{0}, a_{1}=3.06 \lambda_{0}, \varepsilon_{r}=3.25$, $k_{z}=0$ for computation of $\widetilde{G}_{z z}^{E}$ and $k_{z}=k_{s}\left(1+j T_{1}\right)$ for computation of $\widetilde{G}_{\varphi z}^{E}, \widetilde{G}_{\varphi \varphi}^{E}$. As seen in Figures $1-3$, it can be found that results of the spectral domain Green function using (2) converge difficultly even for $N_{t}=300$. Especially for $\widetilde{G}_{\varphi \varphi}^{E}$ shown in Figure 3, the numerical results seem to 
be divergent. But the results of the spectral domain Green function using (5) converge very quickly and one can make $N_{t}=200$ to get accurate results for the spectral domain Green function.

When $k_{z} \rightarrow \infty$, it is found that $C_{u v}\left(k_{z}\right)$ is convergent to $C_{u v}^{\infty}$, so, extracting the asymptotic part of the spatial domain Green function components with respect to $k_{z}$, the expressions for calculating the spatial domain Green function of electric field can be written as

$$
\begin{aligned}
& G_{u v}^{E}=\left(\frac{j \partial}{\partial z}\right)^{e_{3}}\{\left\{\frac{-1}{8 \omega \pi} \int_{-\infty}^{\infty}\left(\tilde{g}_{u v 1}^{E}+\tilde{g}_{u v 2}^{E}\right)\right. \\
&\left.\times \cos \left[k_{z}\left(z-z^{\prime}\right)\right] d k_{z}\right\}+\frac{-1}{8 \omega \pi} C_{u v}^{\infty} F_{2}^{u v}, \\
& \tilde{g}_{u v 1}^{E}= \sum_{n=-\infty}^{\infty}\left(k_{\rho i}^{2}\right)^{e_{1}} n^{e_{2}} H_{n}^{(2)}\left(k_{\rho i} \rho\right) J_{n}\left(k_{\rho i} \rho^{\prime}\right) \\
& \times\left(f^{u v}\left(n, k_{z}\right)-C_{u v}\left(k_{z}\right)\right) e^{j n\left(\varphi-\varphi^{\prime}\right)}, \\
& \tilde{g}_{u v 2}^{E}=\left(C_{u v}\left(k_{z}\right)-C_{u v}^{\infty}\right) F_{1}^{u v}\left[H_{0}^{(2)}\left(k_{\rho i}\left|\rho-\rho^{\prime}\right|\right)\right] .
\end{aligned}
$$

Using the following equations:

$$
\begin{gathered}
I_{1}=\int_{-\infty}^{\infty} H_{0}^{(2)}\left(k_{\rho i}\left|\boldsymbol{\rho}-\boldsymbol{\rho}^{\prime}\right|\right) e^{-j k_{z}\left(z-z^{\prime}\right)}=\frac{2 j e^{-j k_{i}\left|\mathbf{r}-\mathbf{r}^{\prime}\right|}}{\left|\mathbf{r}-\mathbf{r}^{\prime}\right|}, \\
I_{2}=\int_{-\infty}^{\infty} k_{\rho i} \frac{\partial\left[H_{0}^{(2)}\left(k_{\rho i}\left|\boldsymbol{\rho}-\boldsymbol{\rho}^{\prime}\right|\right)\right]}{\partial\left(k_{\rho i}\left|\boldsymbol{\rho}-\boldsymbol{\rho}^{\prime}\right|\right)} e^{-j k_{z}\left(z-z^{\prime}\right)} \\
=2 j\left(-j k_{s}\left|\mathbf{r}-\mathbf{r}^{\prime}\right|-1\right)\left|\boldsymbol{\rho}-\boldsymbol{\rho}^{\prime}\right| \frac{e^{-j k_{s}\left|\mathbf{r}-\mathbf{r}^{\prime}\right|}}{\left|\mathbf{r}-\mathbf{r}^{\prime}\right|^{3}}, \\
I_{3}=\int_{-\infty}^{\infty} k_{\rho i}^{2} \frac{\partial^{2}\left[H_{0}^{(2)}\left(k_{\rho i}\left|\boldsymbol{\rho}-\boldsymbol{\rho}^{\prime}\right|\right)\right]}{\partial\left(k_{\rho i}\left|\boldsymbol{\rho}-\boldsymbol{\rho}^{\prime}\right|\right)^{2}} e^{-j k_{z}\left(z-z^{\prime}\right)} \\
=2 j\left[-k_{s}^{2}\left|\boldsymbol{\rho}-\boldsymbol{\rho}^{\prime}\right|^{2}\left|\mathbf{r}-\mathbf{r}^{\prime}\right|^{2}-\left(z-z^{\prime}\right)^{2}-j\left(z-z^{\prime}\right)^{2}\right. \\
\left.\quad \times k_{s}\left|\mathbf{r}-\mathbf{r}^{\prime}\right|+2\left|\boldsymbol{\rho}-\boldsymbol{\rho}^{\prime}\right|^{2}+2 j\left|\boldsymbol{\rho}-\boldsymbol{\rho}^{\prime}\right|^{2} k_{s}\left|\mathbf{r}-\mathbf{r}^{\prime}\right|\right] \\
\times \frac{e^{-j k_{s}\left|\mathbf{r}-\mathbf{r}^{\prime}\right|}}{\left|\mathbf{r}-\mathbf{r}^{\prime}\right|^{5}} .
\end{gathered}
$$

Expressions for calculating $F_{2}^{u v}$ in (6) can be deducted. Analysing $G_{u v}^{E}$ in (6), one can find that $G_{u v}^{E}$ also includes two parts. The first part is an infinite integral with respect to $k_{z}$ which is now fast decaying with respect to even for very small $\left|\varphi-\varphi^{\prime}\right|$ values and can be solved by the method of deformed integration path proposed in [13]. The second part is the asymptotic part of the spatial domain Green function which has closed form expressions.

2.2. Solution for Axial Line Problem $\left(\left|\rho-\rho^{\prime}\right|=0\right.$ and $\left|\varphi-\varphi^{\prime}\right|<$ $0.05)$. Although a twice-extracting technique is proposed to solve the unaxial line problem in the above section, as to axial line problem, $H_{0}^{(2)}\left(k_{\rho i}\left|\boldsymbol{\rho}-\boldsymbol{\rho}^{\prime}\right|\right)$ in (6) shows more

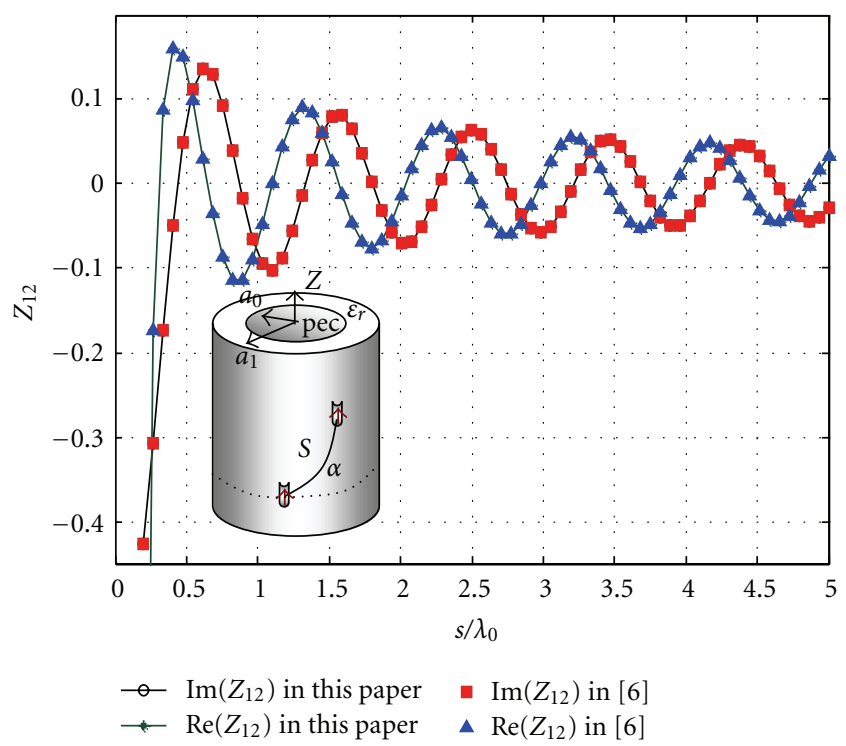

Figure 6: Real and imaginary parts of the mutual impedance $\left(Z_{12}\right)$ between two identical $z$-directed current sources versus separation $S$ when $\alpha=700^{\circ}$ (unaxial line problem).

singular and is also difficult to calculate. Fortunately, [7] does much research on this problem and obtains an approximated representation of spatial Green's function for this case by performing Watson's transformation.

When $S \rightarrow 0(S$ is shown in Figure 6), the method of [7] is not valid because of high-order singular problem in this condition. Even using the asymptotic extraction approach in [15] convergence is still difficult in this case. However, the Green function of planar stratified media can be used instead, because cylindrical stratified media can be treated as planar stratified media approximately when $S<$ $0.2 \lambda_{0}\left(\lambda_{0}\right.$ is the free space wave length). Therefore, to sum up, computation of the spatial Green function for CCMA can be divided into two parts which are shown in Figure 4 . The first part is about the unaxial line problem (defined as $\mid \rho-$ $\rho^{\prime} \mid=0$ and $\left.\left|\varphi-\varphi^{\prime}\right|>0.05\right)$, and the other is about the axial line problem (defined as $\left|\rho-\rho^{\prime}\right|=0$ and $\left|\varphi-\varphi^{\prime}\right|<0.05$ ). The axial line problem involves two subdomains. One is $S>0.2 \lambda_{0}$, and the other is $S<0.2 \lambda_{0}$. The method proposed in [7] is used to attack the problem in $S>0.2 \lambda_{0}$ domain, and the Green function of planar stratified media is used to solve the problem in $S<0.2 \lambda_{0}$ domain. Computation for the Green function of planar stratified media can be found in [14-17].

2.3. Theoretical Analysis of Arbitrary Shape CCMA Fed by Microstripline. Considering CCMA fed by microstripline, $\mathbf{E}_{\rho}^{s}$ does not need to be considered, so one can get

$$
\left[\begin{array}{c}
\mathbf{E}_{\varphi}^{s} \\
\mathbf{E}_{z}^{s}
\end{array}\right]=\left\langle\left[\begin{array}{cc}
\overline{\mathbf{G}}_{\varphi \varphi}^{E} & \overline{\mathbf{G}}_{\varphi z}^{E} \\
\overline{\mathbf{G}}_{z \varphi}^{E} & \overline{\mathbf{G}}_{z z}^{E}
\end{array}\right]\left[\begin{array}{c}
\mathbf{J}_{\varphi} \\
\mathbf{J}_{z}
\end{array}\right]\right\rangle .
$$




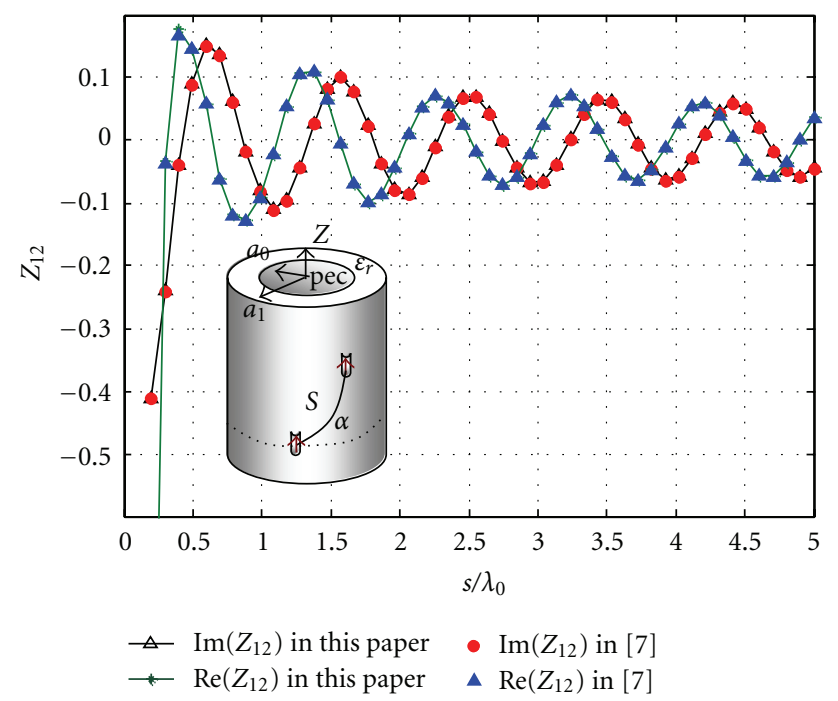

FIgURE 7: Real and imaginary parts of the mutual impedance $\left(Z_{12}\right)$ between two identical $z$-directed current sources versus separation $S$ when $\alpha=90^{\circ}$ (axial line problem).

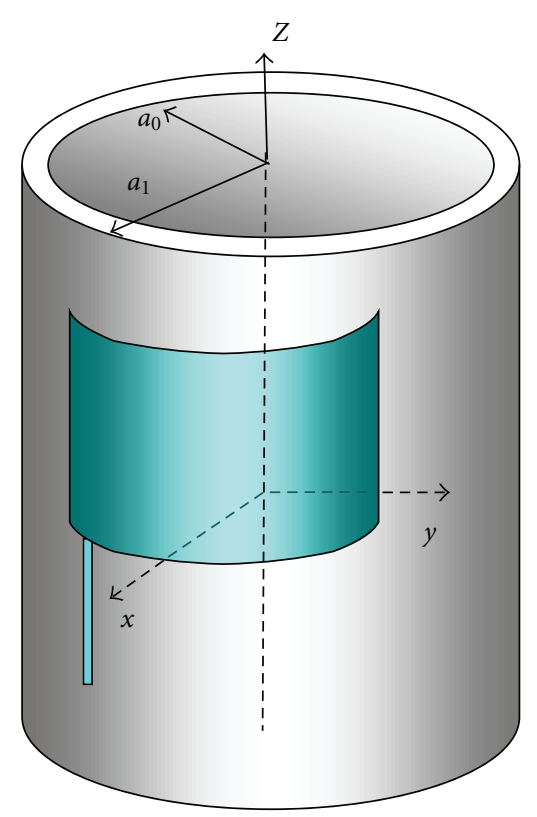

FIgURE 8: CCMA fed by microstripline.

If the source current $\mathbf{J}^{i}=\left[\begin{array}{ll}\mathbf{J}_{\varphi}^{i} & \mathbf{J}_{z}^{i}\end{array}\right]^{T}$ is excited at the end of microstripline as shown in Figure 5, then excited electric field is written as

$$
\left[\begin{array}{c}
\mathbf{E}_{\varphi}^{i} \\
\mathbf{E}_{z}^{i}
\end{array}\right]=\left\langle\left[\begin{array}{cc}
\overline{\mathbf{G}}_{\varphi \varphi}^{E} & \overline{\mathbf{G}}_{\varphi z}^{E} \\
\overline{\mathbf{G}}_{z \varphi}^{E} & \overline{\mathbf{G}}_{z z}^{E}
\end{array}\right]\left[\begin{array}{c}
\mathbf{J}_{\varphi}^{i} \\
\mathbf{J}_{z}^{i}
\end{array}\right]\right\rangle .
$$

Using the Galerkin testing technique, on the patch of CCMA, we arrive at

$$
\left\langle\mathbf{f}^{w},\left.\mathbf{E}^{s}\right|_{t}\right\rangle=-\left\langle\mathbf{f}^{w},\left.\mathbf{E}^{i}\right|_{t}\right\rangle,
$$

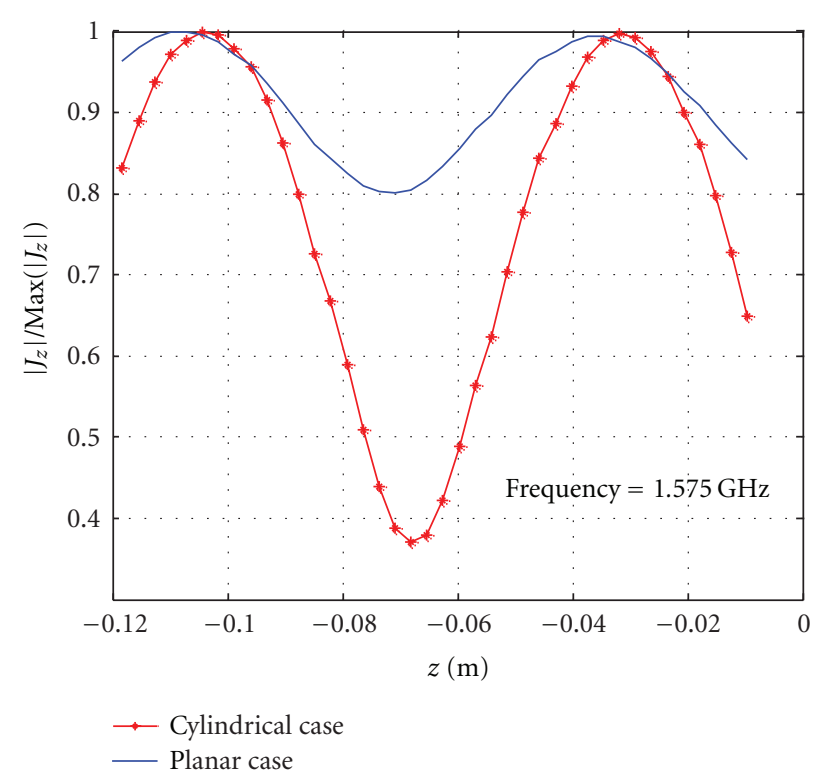

FIGURE 9: Comparison of the current distribution on microstripline.

where $\mathbf{f}^{w}$ is the test basis function in MoM. Upon substituting (8) and (9) into (10), one obtains

$$
\begin{array}{r}
\int_{S} \int_{S^{\prime}}\left[\mathbf{f}_{\varphi}^{w} \cdot\left(\overline{\mathbf{G}}_{\varphi \varphi}^{E} \cdot \mathbf{J}_{\varphi}+\overline{\mathbf{G}}_{\varphi z}^{E} \cdot \mathbf{J}_{z}\right)\right] d S^{\prime} d S \\
=-\int_{S} \int_{S^{i}}\left[\mathbf{f}_{\varphi}^{w} \cdot\left(\overline{\mathbf{G}}_{\varphi \varphi}^{E} \cdot \mathbf{J}_{\varphi}^{i}+\overline{\mathbf{G}}_{\varphi z}^{E} \cdot \mathbf{J}_{z}^{i}\right)\right] d S^{i} d S, \\
\int_{S} \int_{S^{\prime}}\left[\mathbf{f}_{z}^{w} \cdot\left(\overline{\mathbf{G}}_{z \varphi}^{E} \cdot \mathbf{J}_{\varphi}+\overline{\mathbf{G}}_{z z}^{E} \cdot \mathbf{J}_{z}\right)\right] d S^{\prime} d S \\
\quad=-\int_{S} \int_{S^{i}}\left[\mathbf{f}_{z}^{w} \cdot\left(\overline{\mathbf{G}}_{z \varphi}^{E} \cdot \mathbf{J}_{\varphi}^{i}+\overline{\mathbf{G}}_{z z}^{E} \cdot \mathbf{J}_{z}^{i}\right)\right] d S^{i} d S .
\end{array}
$$

The current distribution on the patch of the antenna can be approximated in terms of $\mathbf{f}_{n}$ as

$$
\mathbf{J}_{s}=\sum_{n} I_{n} \mathbf{f}_{n}
$$

where $\mathbf{f}_{n}$ is the RWG basis function in this paper, which is different from the traditional method in [8-10], and one can find the define about RWG in [18]. From (11) and (12), if $\mathbf{f}^{w}$ is equal to $\mathbf{f}_{m}$, current coefficient $I_{n}$ can be gotten from the following identity:

$$
\begin{aligned}
& I_{n} \int_{S} \int_{S^{\prime}}\left[\begin{array}{ll}
\left.\mathbf{f}_{m}\right|_{\varphi} & \left.\mathbf{f}_{m}\right|_{z}
\end{array}\right] \cdot\left[\begin{array}{cc}
\overline{\mathbf{G}}_{\varphi \varphi}^{E} & \overline{\mathbf{G}}_{\varphi z}^{E} \\
\overline{\mathbf{G}}_{z \varphi}^{E} & \overline{\mathbf{G}}_{z z}^{E}
\end{array}\right]\left[\begin{array}{c}
\left.\mathbf{f}_{n}\right|_{\varphi} \\
\left.\mathbf{f}_{n}\right|_{z}
\end{array}\right] d S^{\prime} d S \\
& =-\int_{S} \int_{S^{i}}\left[\begin{array}{ll}
\left.\mathbf{f}_{m}\right|_{\varphi} & \left.\mathbf{f}_{m}\right|_{z}
\end{array}\right] \cdot\left[\begin{array}{cc}
\overline{\mathbf{G}}_{\varphi \varphi}^{E} & \overline{\mathbf{G}}_{\varphi z}^{E} \\
\overline{\mathbf{G}}_{z \varphi}^{E} & \overline{\mathbf{G}}_{z z}^{E}
\end{array}\right]\left[\begin{array}{c}
\mathbf{J}_{\varphi}^{i} \\
\mathbf{J}_{z}^{i}
\end{array}\right] d S^{i} d S .
\end{aligned}
$$


Considering $m, n=1,2, \ldots N$, formula (13) can be written in matrix form as

$$
\left[\begin{array}{cccc}
\mathbf{Z}_{11} & \mathbf{Z}_{12} & \cdots & \mathbf{Z}_{1 N} \\
\mathbf{Z}_{21} & \mathbf{Z}_{22} & \cdots & \mathbf{Z}_{2 N} \\
\vdots & \vdots & \vdots & \vdots \\
\mathbf{Z}_{N 1} & \mathbf{Z}_{N 2} & \cdots & \mathbf{Z}_{N N}
\end{array}\right]\left[\begin{array}{c}
\mathbf{I}_{1} \\
\mathbf{I}_{2} \\
\vdots \\
\mathbf{I}_{N}
\end{array}\right]=\left[\begin{array}{c}
\mathbf{V}_{1} \\
\mathbf{V}_{2} \\
\vdots \\
\mathbf{V}_{N}
\end{array}\right]
$$

where

$$
\begin{gathered}
\mathbf{Z}_{m n}=\int_{S} \int_{S^{\prime}}\left[\begin{array}{ll}
\left.\mathbf{f}_{m}\right|_{\varphi} & \left.\mathbf{f}_{m}\right|_{z}
\end{array}\right] \\
\cdot\left[\begin{array}{cc}
\overline{\mathbf{G}}_{\varphi \varphi}^{E} & \overline{\mathbf{G}}_{\varphi z}^{E} \\
\overline{\mathbf{G}}_{z \varphi}^{E} & \overline{\mathbf{G}}_{z z}^{E}
\end{array}\right]\left[\begin{array}{c}
\left.\mathbf{f}_{n}\right|_{\varphi} \\
\left.\mathbf{f}_{n}\right|_{z}
\end{array}\right] d S^{\prime} d S, \\
\mathbf{V}_{m}=-\int_{S} \int_{S^{i}}\left[\begin{array}{cc}
\left.\mathbf{f}_{m}\right|_{\varphi} & \left.\mathbf{f}_{m}\right|_{z}
\end{array}\right] \\
\cdot\left[\begin{array}{cc}
\overline{\mathbf{G}}_{\varphi \varphi}^{E} & \overline{\mathbf{G}}_{\varphi z}^{E} \\
\overline{\mathbf{G}}_{z \varphi}^{E} & \overline{\mathbf{G}}_{z z}^{E}
\end{array}\right]\left[\begin{array}{c}
\mathbf{J}_{\varphi}^{i} \\
\mathbf{J}_{z}^{i}
\end{array}\right] d S^{i} d S .
\end{gathered}
$$

In order to calculate $\mathbf{V}_{m}$ in (15), $\mathbf{J}^{i}$ can be treated as a halfRWG basis function added at the end of the microstripline which is shown in Figure 5.

After getting all the current coefficients in (14), one can obtain the current distributions on the patch and microstripline. Using GPOF method [19], current distributions on the microstripline can be expressed as

$$
I(z) \approx \sum_{i=1}^{K} I_{i} e^{\left(\alpha_{i}+j \beta_{i}\right) z},
$$

where $I_{i}, \alpha_{i}$, and $\beta_{i}$ are parameters approximated by GPOF method. It is found that $\alpha_{i} \ll \beta_{i}$, so (16) can be approximated as follows:

$$
I(z)=I_{1} e^{-j \beta_{1} z}+I_{2} e^{j \beta_{1} z} \cdots I_{K} e^{j \beta_{K} z} .
$$

In (17), $I_{1}$ and $I_{2}$ represent the two opposite travelling waves $I^{+}$and $I^{-}$. The others $\left(I_{3}, I_{4}, \ldots I_{K}\right)$ are the highorder travelling waves which can be ignored generally. So the reflection coefficient can be found as

$$
\begin{gathered}
S_{11}=\frac{I_{2}}{I_{1}}, \\
\mathrm{~dB}\left(S_{11}\right)=20 \log 10\left|\frac{I_{2}}{I_{1}}\right|,
\end{gathered}
$$

and the impedance of the antenna is given by

$$
Z_{\text {in }}=\frac{1+S_{11}}{1-S_{11}} Z_{c}
$$

where $Z_{c}$ is the characteristic impedance of microstripline. In addition, to calculate the far field of the CCMA fed by microstripline, the method in [19] can be used. However, it should be noticed that this paper chooses the RWG basis function to approximate the current, so formulas for calculating current in [19] should be changed properly.

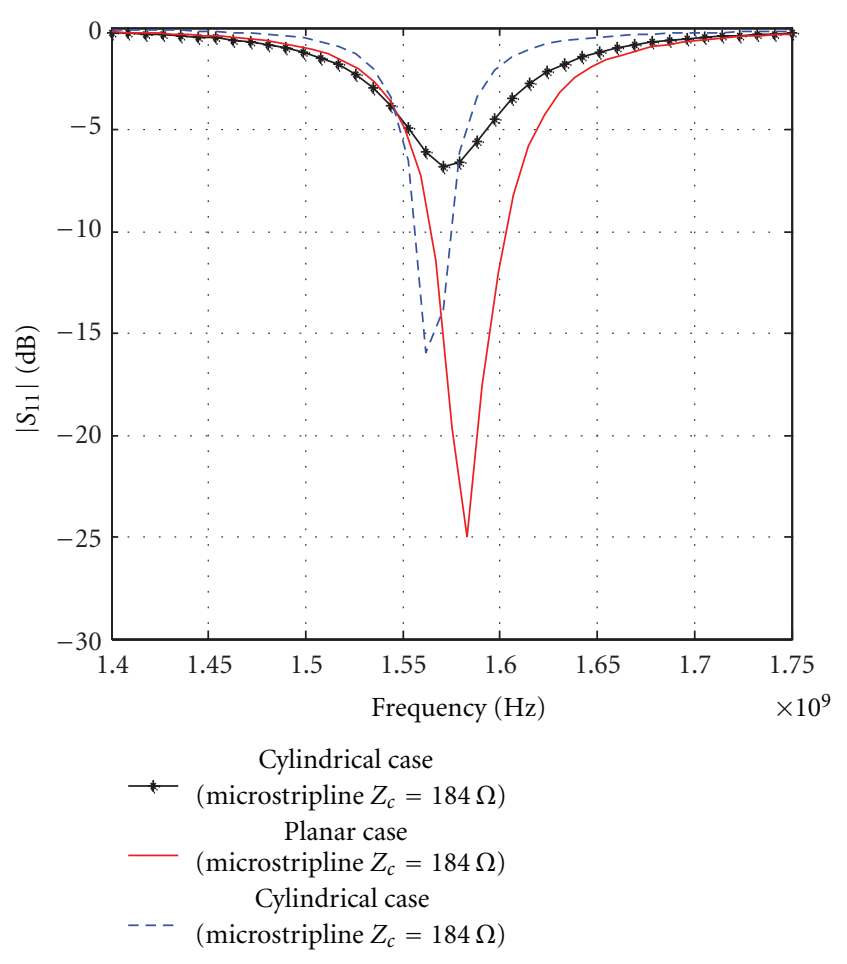

Figure 10: Comparison of the reflection coefficient.

\section{Numerical Results}

3.1. Mutual Impedance between the Current Modes. In order to assess the validity and accuracy of the method proposed in this paper, numerical results for the mutual impedance between two tangential electric current modes are obtained using the domain-division solution and compared with the results of $[6,7]$. The numerical results are shown in Figures 6 and 7, and the parameters of the cylinder and substrate in these figures are set as $a_{0}=3 \lambda_{0}, a_{1}=3.06 \lambda_{0}, \varepsilon_{r}=3.25$. Figure 6 shows that results obtained by the twice-extracting technique in this paper are in good agreement with the results in [6], so the technique is effective for the unaxial line problem. As seen in Figure 7, the method of [7] is used successfully to solve axial line problem in this paper.

3.2. Analysis of a CCMA Fed by Microstripline. Using the method in this paper, a CCMA fed by microstripline illustrated in Figure 8 is analysed. Sizes of the antenna are set as $\varphi$ - and $z$-direction lengths of patch are $0.0615 \mathrm{~m}$ and 0.0604 , respectively, microstripline length is $0.1278 \mathrm{~m}$, the feed point is $0.02696 \mathrm{~m}$ away from the bottom edge's centre of the patch in $\varphi$-direction, thickness of the substrate is $0.00234 \mathrm{~m}$, the microstripline wide is $0.1 \mathrm{~mm}\left(Z_{c}=184\right)$, $\varepsilon_{r}=2.32$, and $a_{0}=0.187451 \mathrm{~m}$. Results of the normalised current distribution on microstripline, reflection coefficient, and normalised radiation patterns are presented in Figures 9-12.

Figures 9 and 10 show that resonance frequency of the antenna in planar case is $1.575 \mathrm{GH}$, but in cylindrical case, the reflection coefficient does not satisfy the request 


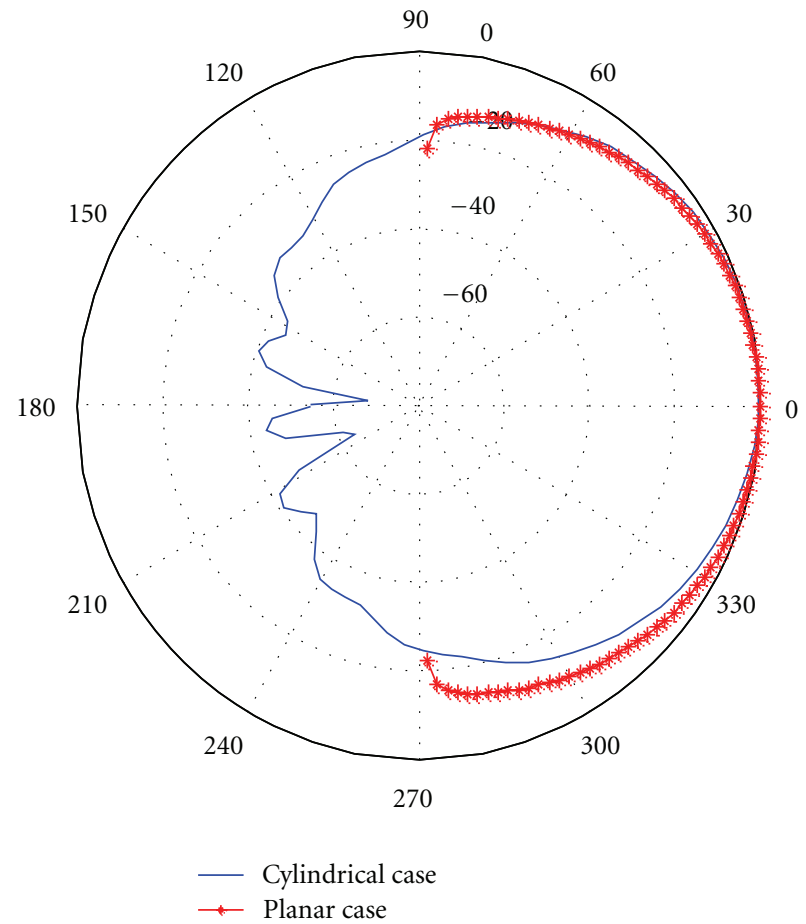

Figure 11: Normalised radiation field in $x o y$-plane (dB).

$\mathrm{dB}\left(S_{11}\right)<-10 \mathrm{~dB}$ at $1.575 \mathrm{GHz}$. The reason for it may be that characteristic impedance of the microstripline in cylindrical case does not match the impedance of the antenna. So the sizes of microstripline should be designed again to match the impedance of the CCMA. If the microstripline wide is designed to be $2.1 \mathrm{~mm}\left(Z_{c}=71 \Omega\right)$, then the reflection coefficient of the CCMA will decrease, which is shown in Figure 10.

Figures 11 and 12 show that backward radiation field appears in cylindrical case, but the frontward radiation field is similar to the planar case.

\section{Conclusion}

The development of the researches on CCMA is unmatured, and the Green functions of cylindrically stratified media are very difficult to calculate accurately and efficiently. Based on the former researches [1-14], this paper proposes a domaindivision solution to solve Hankel function's singular problem of Sommerfeld integral in calculating the spatial domain Green function of cylindrically stratified media and realize the computation for all elements of $Z$-matrix in MoM. Then, theoretical analysis of arbitrary shape CCMA fed by microstripline is done by MoM with the RWG basis function which is more general than the rooftop basis function used in the traditional method. At last, numerical results in the form of mutual impedance between two current modes reveal that the proposed method is valid and efficient. In addition, comparison of the results of a CCMA and a planar microstrip antenna fed by microstripline depicts that the current distribution on microstripline, reflection coefficient, and

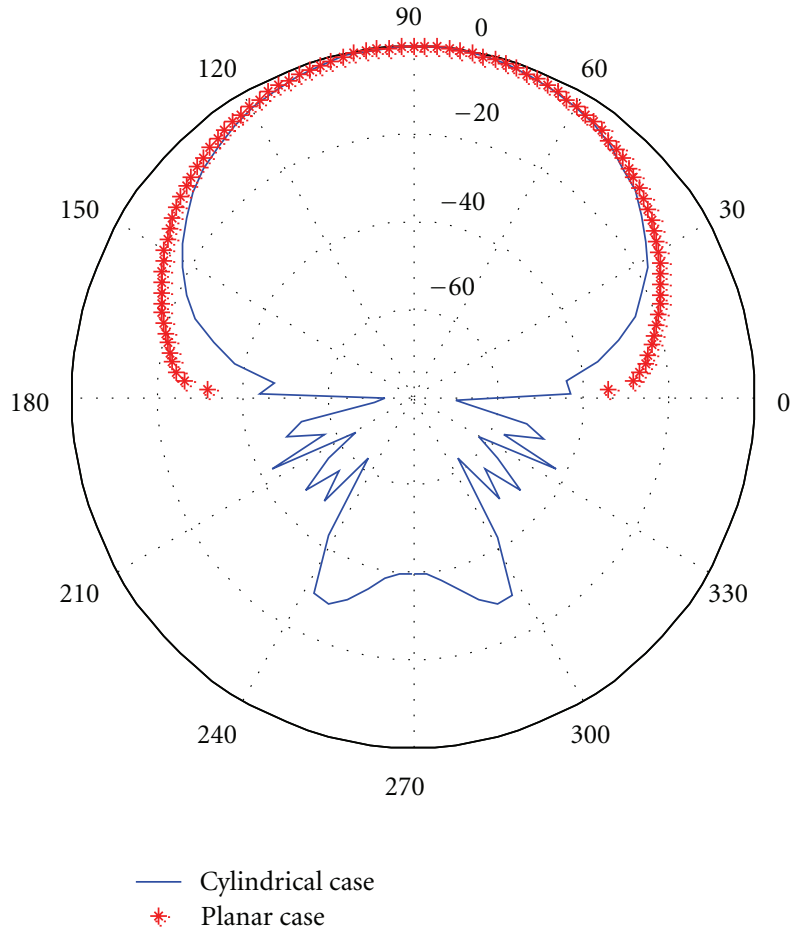

FIGURE 12: Normalised radiation field in xoz-plane (dB).

normalised radiation patterns are affected by antenna patch's curvature. It also shows that, comparing with the planar counterpart microstrip antenna, the reflection coefficient of the CCMA increases, the current on the microstripline varies sharply, and the backward radiation field appears. However, the frontward radiation field is similar to the planar case. In order to improve the performance of the CCMA, the sizes of microstripline should be designed properly to match the impedance of CCMA.

\section{References}

[1] C. Y. Huang, Y. H. Liu, and K. L. Wong, "Input impedance calculation of cylindrical rectangular microstrip antennas using GTLM theory," in Proceedings of the IEEE Antennas and Propagation Society International Symposium, pp. 1792-1795, June 1995.

[2] M. V. T. Heckler, M. Bonadiman, R. Schildberg, L. Cividanes, and J. C. D. S. Lacava, "CAD Package to Design Rectangular Probe-Fed Microstrip Antennas Conformed on Cylindrical Structures," in Proceedings of the SMBO/IEEE MTT-S International Microwave and Optoelectronics Conference (IMOC '03), pp. 747-752, September 2003.

[3] M. V. T. Heckler, M. Bonadiman, J. C. D. S. Lacava, and L. Cividanes, "Analysis of cylindrical circumferential array with circular polarization for space applications," in Proceedings of the IEEE Antennas and Propagation Society Symposium, pp. 117-120, June 2004.

[4] J. Sun, C. F. Wang, L. W. Li, and M. S. Leong, "Further improvement for fast computation of mixed potential Green's functions for cylindrically stratified media," IEEE Transactions on Antennas and Propagation, vol. 52, no. 11, pp. 3026-3036, 2004. 
[5] S. Karan, V. B. Ertürk, and A. Altintas, "Closed-form Green's function representations in cylindrically stratified media for method of moments applications," IEEE Transactions on Antennas and Propagation, vol. 57, no. 4, pp. 1158-1168, 2009.

[6] V. B. Ertürk and R. G. Rojas, "Efficient computation of surface fields excited on a dielectric-coated circular cylinder," IEEE Transactions on Antennas and Propagation, vol. 48, no. 10, pp. 1507-1516, 2000.

[7] V. B. Ertürk and R. G. Rojas, "Paraxial space-domain formulation for surface fields on a large dielectric coated circular cylinder," IEEE Transactions on Antennas and Propagation, vol. 50, no. 11, pp. 1577-1587, 2002.

[8] V. B. Ertürk and R. G. Rojas, "Efficient analysis of input impedance and mutual coupling of microstrip antennas mounted on large coated cylinders," IEEE Transactions on Antennas and Propagation, vol. 51, no. 4, pp. 739-749, 2003.

[9] T. N. Kaifas and J. N. Sahalos, "Analysis of printed antennas mounted on a coated circular cylinder of arbitrary size," IEEE Transactions on Antennas and Propagation, vol. 54, no. 10, pp. 2797-2807, 2006.

[10] M. He and X. Xu, "Closed-form solutions for analysis of cylindrically conformal microstrip antennas with arbitrary radii," IEEE Transactions on Antennas and Propagation, vol. 53, no. 1, pp. 518-525, 2005.

[11] A. Svezhentsev and G. A. E. Vandenbosch, "Efficient spatial domain moment method solution of cylindrical rectangular microstrip antennas," IEE Proceedings, vol. 153, no. 4, pp. 376384, 2006.

[12] R. C. Acar and G. Dural, "Mutual coupling of printed elements on a cylindrically layered structure using closed-form green's functions," Progress in Electromagnetics Research, vol. 78, pp. 103-127, 2008.

[13] C. Tokgöz and G. Durai, "Closed-formgreen's functions for cylindrically stratified media," IEEE Transactions on Microwave Theory and Techniques, vol. 48, no. 1, pp. 40-49, 2000.

[14] W. C. Chew, Waves and Fields in Inhomogeneous Media, Van Nostrand, New York, NY, USA, 1995.

[15] J. Wu, S. K. Khamas, and G. G. Cook, "An efficient asymptotic extraction approach for the green's functions of conformal antennas in multilayered cylindrical media," IEEE Transactions on Antennas and Propagation, vol. 58, no. 11, pp. 3737-3742, 2010.

[16] K. A. Michalski and D. Zheng, "Electromagnetic scattering and radiation by surfaces of arbitrary shape in layered media-I: theory," IEEE Transactions on Antennas and Propagation, vol. 38, no. 3, pp. 335-344, 1990.

[17] K. A. Michalski and D. Zheng, "Electromagnetic scattering and radiation by surfaces of arbitrary shape in layered media-II: implementation and results for contiguous halfspaces," IEEE Transactions on Antennas and Propagation, vol. 38, no. 3, pp. 345-352, 1990.

[18] S. Makarov, "MoM antenna simulations with Matlab: RWG basis functions," IEEE Antennas and Propagation Magazine, vol. 43, no. 5, pp. 100-107, 2001.

[19] K. Tapan and O. Pereira, "Using the matrix pencil method to estimate the parameters of a sum of complex exponentials," IEEE Antennas and Propagation Magazine, vol. 37, no. 1, pp. 48-55, 1995. 

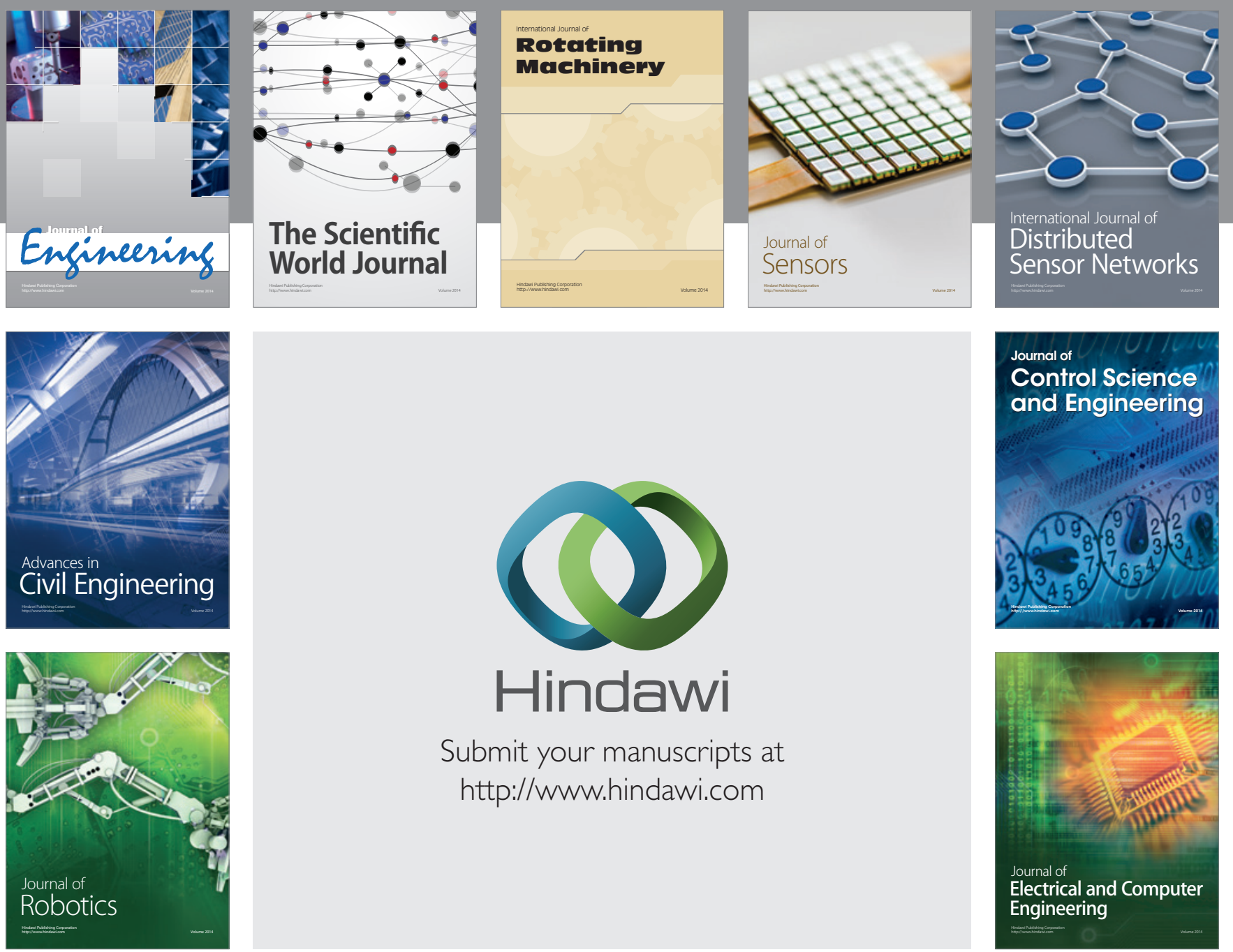

Submit your manuscripts at

http://www.hindawi.com
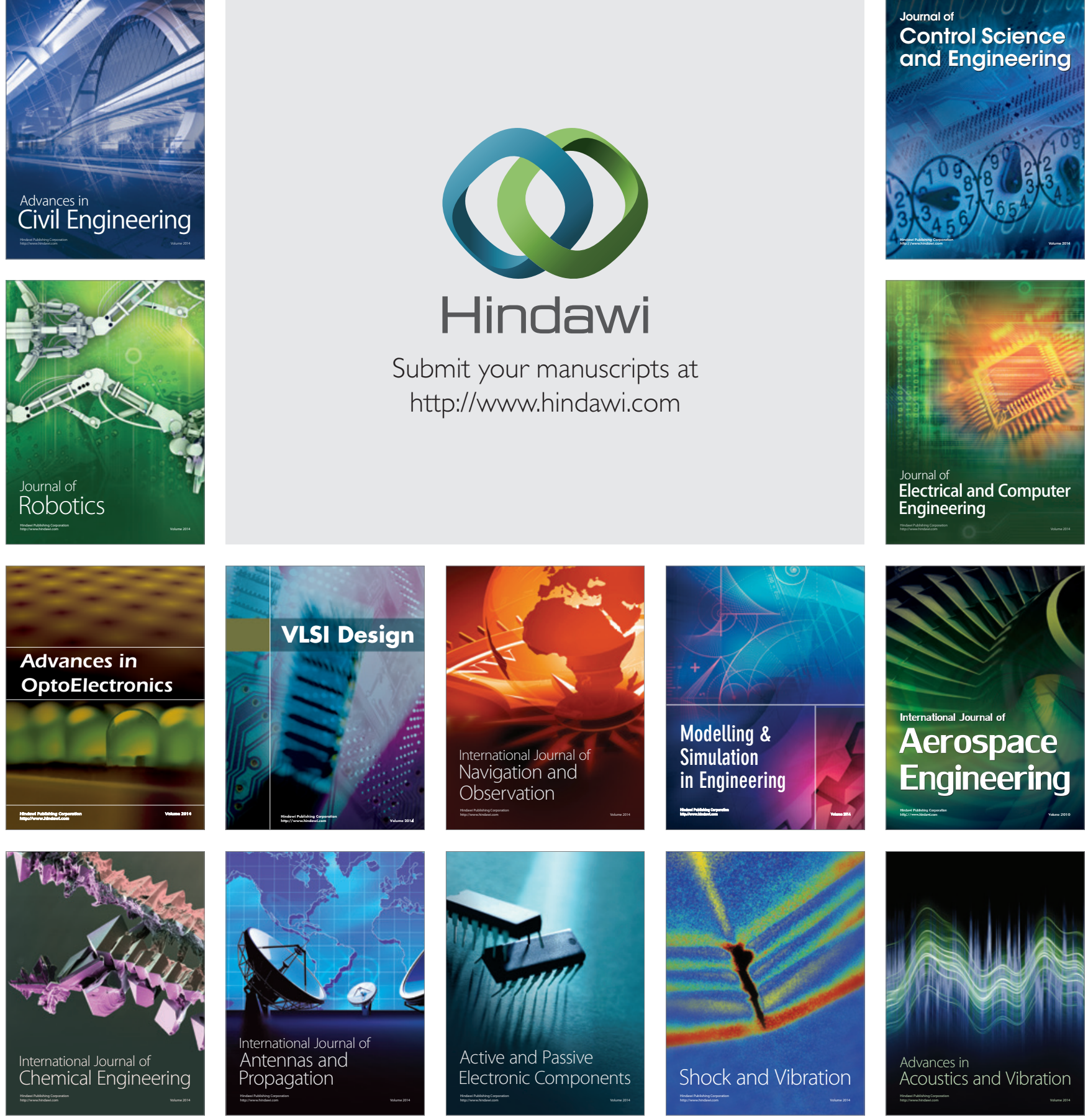